\title{
Relevancia y prevalencia del estrés post-traumático post-terremoto como problema de salud pública en Constitución, Chile
}

\author{
The relevance and prevalence of post-traumatic stress after an \\ earthquake: public health problem in Constitucion, Chile
}

\author{
Marcelo Leiva-Bianchi \\ Facultad de Psicología, Universidad de Talca. Talca, Chile. marcleiva@utalca.cl \\ Recibido 12 Enero 2011/Enviado para Modificación 1 Agosto 2011/Aceptado 17 Agosto 2011

\section{RESUMEN}

Objetivos Según investigaciones previas, después del terremoto y tsunami del 27 de febrero de $2010(27-\mathrm{F})$, se espera un aumento de la prevalencia del estrés posttraumático (TEPT). Esta investigación pretende mostrar la importancia de este problema, identificando la prevalencia del TEPT en la ciudad de Constitución, una de las más afectadas por el 27-F.

Método Mediante la escala Short Posttraumatic Stress Disorder Rating Interview (SPRINT-E), se midió la cantidad de síntomas intensos de TEPT en dos muestras: apoderados de un colegio municipal y personal de la red de atención primaria.

Resultados La prevalencia del TEPT en ambos grupos es mayor a lo esperado: $36 \%$ en el grupo de apoderados (en el cual se esperaba un $23 \%$ ) y $20 \%$ en el de funcionarios (en el cual se esperaba un $15 \%$ ).

Conclusiones Se reflexiona respecto del grave problema de salud mental pública presente en esta ciudad en particular y en todas las zonas afectadas en general. También se discute respecto de la factibilidad de resolver este problema de salud pública, mediante los recursos disponibles en la red de salud. Si bien su resolución es compleja, es posible apoyar iniciativas científicas de investigación aplicada, como la que actualmente está llevando a cabo la Facultad de Psicología de la Universidad de Talca.

Palabras Clave: Trastorno por estrés postraumático, prevalencia, salud mental, salud pública, terremotos, tsunamis (fuente: DeCS, BIREME).

\section{ABSTRACT}

Objectives An increase in the prevalence of post-traumatic stress disorder (PTSD) is expected following the February $27^{\text {th }} 2010$ (F-27) earthquake and tsunami, according to previous research; the current research was aimed at showing the 
importance of this problem by identifying PTSD prevalence in Constitucion, one of the Chilean cities most affected by F-27.

Methods The number of severe PTSD symptoms was measured in two samples taken from staff from a local school and a primary care network, using the short posttraumatic stress disorder rating interview (SPRINT-E) scale.

Results PTSD prevalence was higher than expected in both groups: $36 \%$ in the local school staff (where $23 \%$ was expected) and $20 \%$ for primary care network staff ( $15 \%$ was expected).

Conclusions The article deals with PTSD prevalence, reflecting on the serious public mental health problem affecting this city in particular and all affected areas in general. It also discusses the feasibility of solving this public health problem by using available health network resources. While its resolution is complex, initiatives in applied research can always be supported, such as those currently being conducted by the University of Talca's psychology faculty.

Key Words: Stress disorder, post-traumatic, prevalence, mental health, public health, earthquake, tsunami (source: $M e S H, N L M$ ).

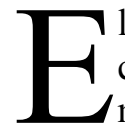
1 sábado 27 de febrero de 2010 a las 03 horas y 34 minutos hora continental chilena, se produjo un terremoto como pocos en la historia mundial. Su duración (3 minutos), su magnitud ( 8,8 grados Richter, la sexta mayor registrada en el mundo desde inicios del siglo XX) y la ubicación de su epicentro $\left(35,9^{\circ}\right.$ latitud sury $72,7^{\circ}$ longitud oeste, 335 kilómetros al suroeste de Santiago, la zona que concentra más población en Chile) así lo confirman (1). Además, minutos después, el terremoto produjo un tsunami que azotó las costas de la zona central entre las regiones del Libertador Bernardo O’Higgins hasta la del Bío-Bío, siendo especialmente destructivo en las costas de la Región del Maule. Dada la cantidad de víctimas y damnificados fue esta última región la que recibió el mayor impacto de ambos eventos combinados (2). De hecho, en Constitución, principal ciudad costera de esta región, media hora después del terremoto, tres olas de 8,10 y 8 metros respectivamente, ingresaron por la desembocadura del río Maule y devastaron la ciudad (3).

En vista de estos hechos, resulta evidente el alto impacto del terremoto y el tsunami del 27-F en las personas. Más allá de afectar directamente a la zona más poblada del país (4), del costo en infraestructura (500 mil viviendas presentan daños severos que dejan cerca de 2 millones de personas damnificadas) (3) y en la vida de las personas (521 víctimas fatales y 56 personas desaparecidas) $(5,6)$, es altamente probable que el $27-\mathrm{F}$ tenga también un alto impacto en la salud mental de la población (7). Y, dicho impacto, se traducirá en el aumento de los síntomas del estrés post-traumático (TEPT), uno de los trastornos más característicos después de este tipo de eventos $(8,9)$. 
Según el DSM-IV el TEPT es un tipo de trastorno de ansiedad caracterizado por la aparición de síntomas que siguen a la exposición ya sea directa o indirecta (escuchar narraciones o ver imágenes) a un evento estresante y extremadamente traumático (muertes, amenazas a la integridad física, etc.). La persona responde con temor, desesperanza u horror intensos. El acontecimiento traumático es reexperimentado a través de recuerdos o sueños recurrentes e incontrolables (intrusos) que provocan malestar y en los que se incluyen imágenes, pensamientos o percepciones. Esto produce un malestar psicológico intenso asociado al evitar continuamente recordar lo vivido, al embotamiento (debilitamiento) conductual, y a respuesta fisiológicas de activación; estas respuestas aparecen especialmente cuando la persona se expone a estímulos internos o externos que simbolizan o recuerdan un aspecto del acontecimiento traumático. La activación fisiológica es tal que produce dificultades para conciliar o mantener el sueño, irritabilidad, ataques de ira, dificultades para concentrarse, hipervigilancia y respuestas exageradas de sobresalto. Todas estas alteraciones se prolongan por más de 1 mes y provocan malestar clínico significativo o deterioro social, laboral o de otras áreas importantes de la actividad del individuo (10).

Si bien existe claridad respecto de cuál es la prevalencia del TEPT antes del 27-F (4,4\%) (11), no es posible establecerla con exactitud después de una catástrofe como esta. Por ejemplo, tras los terremotos de 1999 en Taiwán y Grecia se encontraron prevalencias de 4,4\% (12) y 4,5\% (13) respectivamente. Tras el terremoto de California en 1994 (14) y el tsunami asiático del 2004 (15), la prevalencia del TEPT fue de un $42 \%$. En todo caso, diversos estudios sitúan la prevalencia de este trastorno después de un terremoto entre un 10,3\% y un $30 \%$ (16-19). Y, respecto de la prevalencia del TEPT después de un tsunami, se encontró que esta fue de un 70,7 \% en niños del sur de la India (20).

Sea cual sea el estudio, lo esperable es que los síntomas del TEPT aumenten después de la catástrofe del 27-F. De hecho, esperamos que entre un $30 \% \mathrm{y}$ un $40 \%$ de las personas damnificadas del país y entre un $10 \%$ y un $20 \%$ del personal de atención de salud vinculado a labores de emergencia presenten TEPT después del 27-F (21). Y, lo que es aún más grave, un tercio de los pacientes con TEPT continuará con síntomas diez años después del evento traumático, lo cual traerá consecuencias en su vida personal y laboral; incluso, en aquellos casos en que se dé en combinación con otros trastornos como la depresión, puede traer consecuencias gravísimas como el suicidio $(22,23)$. 
En vista de lo anterior, el presente estudio tiene como finalidad conocer cuál es la cantidad de síntomas del TEPT presentes en personas que vivieron el 27-F. Específicamente evaluamos una muestra de personas pertenecientes a la ciudad de Constitución, una de las más afectadas por el terremoto y el tsunami del 27-F.

\section{METODOLOGÍA}

\section{Participantes}

Se evalúa a 107 apoderados de un colegio municipal y a 79 funcionarios de la red de atención primaria, ambos grupos residentes en la ciudad de Constitución al momento de la evaluación y del 27-F. En ambos casos, el muestreo fue intencionado y por conveniencia. Es decir, se eligió la muestra de apoderados por ser representativa de la población general afectada por terremoto y tsunami; y la del personal de la red de atención primaria por ser representativa de un grupo vulnerable porque, además de estar expuesto a ambos eventos, trabajó atendiendo personas afectadas durante y después del 27-F. Además, los participantes decidieron voluntariamente contestar la escala.

\section{Instrumentos}

Para medir el grado de TEPT utilizamos el Short Posttraumatic Stress Disorder Rating Interview (SPRINT-E), instrumento de 11 preguntas diseñado para detectar de manera rápida síntomas de este trastorno en poblaciones afectadas y utilizado después de catástrofes tales como el atentado a las Torres Gemelas en Nueva York y la del huracán Katrina en Nueva Orleans (24-27). Cada pregunta se responde según una escala de intensidad del síntoma medido que va de 0 (mínima intensidad) a 4 (máxima intensidad). Si la respuesta a una pregunta es mayor o igual a 3 puntos, la consideraremos como intensa, tal y como lo recomiendan las creadoras de la escala (26); 3 o más respuestas intensa es un indicador de presencia de TEPT aunque con 7 o más respuestas estaremos seguros de que la probabilidad de que ocurra un falso positivo es muy baja (26).

Procedimientos y análisis de datos

Para aplicar el SPRINT-E en ambas muestras aprovechamos las instancias formales de reuniones mensuales que tanto apoderados como funcionarios tienen (reunión de apoderados en el primer grupo y capacitación en el segundo). Los datos obtenidos de esas evaluaciones fueron sometidos a un análisis estadístico descriptivo con el fin de encontrar la cantidad de síntomas 
intensos experimentados por ambos grupos. Realizamos los análisis mediante el programa SPSS versión 15.

\section{RESULTADOS}

Respecto de las medidas de tendencia central de los síntomas de TEPT en la muestra de apoderados del colegio municipal, encontramos que la media de reacciones intensas fue de 3,3 y la mediana de 3. Por otra parte, la varianza y la desviación típica fueron de 9,4 y 3,1 respectivamente. En cuanto a la forma de la distribución tenemos que su curtosis es negativa $(\mathrm{k}=-0,7$; et $=0,5)$ y su asimetría positiva ( $\mathrm{s}=0,5$; et $=0,2)$; esto indica que las puntuaciones se agrupan menos que la distribución normal en torno a los valores inferiores a la media y, por lo tanto, no se distribuyen normalmente $(\mathrm{KS}=0,2 ; \mathrm{p}<, 01)$.

En cuanto a la frecuencia de los síntomas intensos de TEPT en la muestra de apoderados, tenemos que un 54,2 \% presenta al menos 3 síntomas intensos, mientras que un $17,8 \%$ presenta al menos 7 síntomas intensos. La media entre ambos porcentajes fue de $35,9 \%$.

Ahora bien, respecto de las medidas de tendencia central de las reacciones intensas de TEPT en la muestra de funcionarios de la red de asistencia primaria, encontramos que la media fue de 1,9 y la mediana de 1. Por otra parte, la varianza y la desviación típica fueron de 6,7 y 2,6 respectivamente. En cuanto a la forma de la distribución tenemos que tanto la curtosis $(\mathrm{k}=9,2 ; \mathrm{et}=1,3)$ como la asimetría $(\mathrm{s}=3,0$; et $=0,7)$ son positivas; esto indica que las puntuaciones se agrupan más que lo normal en torno a los valores inferiores a la media $\mathrm{y}$, por ende, no se distribuyen normalmente $(\mathrm{KS}=0,2 ; \mathrm{p}<, 01)$.

En cuanto a la frecuencia de los síntomas intensos de TEPT en la muestra de funcionarios, tenemos que un 32,9\% presenta al menos 3 síntomas intensos, mientras que un $6,3 \%$ presenta al menos 7 síntomas intensos. La media entre ambos porcentajes corresponde a un $19,7 \%$.

\section{DISCUSIÓN}

Inicialmente, tanto en la muestra de apoderados del colegio como en la de funcionarios de la red de salud encontramos una proporción de casos de TEPT superior a la encontrada en otros estudios. Entre los apoderados encontramos entre un $18 \%$ y un $54 \%$ cuando esperábamos encontrar entre un $4 \%$ y un $42 \%$ de casos de TEPT (12-15). Mientras que entre los funcionarios de la red de salud expuestos a 
la catástrofe encontramos entre un $6 \%$ y un $33 \%$ cuando esperábamos encontrar entre un $10 \%$ y un $20 \%$ (21). Esta mayor proporción de síntomas puede deberse a las características del evento vivido por las personas evaluadas (8); ambos grupos estuvieron expuestos al evento doble terremoto y tsunami, lo cual es un factor que aumenta el impacto en las personas y la cantidad de síntomas de trastornos de ansiedad tales como los de la crisis de pánico $(7,28)$. Entonces, el tsunami amplifica el efecto negativo en la salud mental de la población.

La mayor proporción de síntomas de TEPT y el efecto amplificador del tsunami nos hace reflexionar sobre la gravedad del problema de salud mental pública en esta zona en particular y en todas las zonas afectadas en general. Ya conocemos tanto teórica como empíricamente el efecto que la catástrofe del 27-F tuvo en la población y sabemos que es mayor a lo que esperábamos. Sin embargo, no queda claro si este problema ha sido dimensionado por los diversos actores de la red de salud. Una característica contextual importante para hacerse cargo del efecto psicosocial de una catástrofe es todo lo relacionado con el sistema de salud. Para que este sistema sea capaz de reaccionar eficientemente ante situaciones de catástrofe, debe cumplir con las siguientes etapas de acción $(21,29)$ : 1. Efectuar una cuidadosa campaña de contención social, psicoeducación y promoción de higiene mental a través de medios escritos, audiovisuales e Internet; 2. Organizar una amplia operación de campo, con despliegue de equipos psicosociales permanentes en campamentos y albergues, para que realicen primeros auxilios psicológicos y monitorizar la salud mental in situ; 3. Capacitar al personal de salud de los Servicios de Urgencia y Atención Primaria para el diagnóstico, tratamiento inicial y derivación de los pacientes con secuelas psicológicas, ya que sabemos que éstos últimos no consultan espontáneamente; 4. Capacitar a psicólogos y psiquiatras de las regiones más devastadas en la aplicación de psicoterapias centradas en el trauma (terapia cognitivo conductual y terapia EMDR), ya que otros tipos de tratamientos (psicoterapia de apoyo, psicoterapia psicoanalítica, hipnosis o "debriefing") no han demostrado ser efectivos en desastres y, peor aún, podrían ser perjudiciales.

Difícil labor, en vista de las limitaciones de nuestra red de salud mental tanto pública como privada. En términos generales, la red no está preparada para resolver las secuelas psicológicas de esta catástrofe (21). De hecho, si la cantidad de profesionales capacitados es insuficiente para satisfacer la demanda normal de tratamientos de salud mental, con mayor razón aún lo será durante los 4 a 5 años después del 27-F en los cuales se espera una incidencia del TEPT 7,5 veces mayor que en un año normal (30,31). Cabe destacar que la reconstrucción plantea la necesidad de racionalizar los recursos y dar prioridad a las necesidades 
más básicas de la población, intentando también abarcar necesidades de contención y tratamiento psicológico. Por ejemplo, en Constitución, el Hospital ha contratado a un psiquiatra y un psicólogo, por 44 horas mensuales cada uno, mientras que el CESFAM de la misma ciudad posee un equipo de salud mental compuesto por 10 profesionales a jornada parcial. Esto, sin embargo, no es suficiente y el panorama puede ser peor aún en otras zonas afectadas. Otro ejemplo es el de Pelluhue, en donde el equipo de salud mental está compuesto sólo por dos personas. Estos equipos tendrán que atender las necesidades de una población de personas con una cantidad de síntomas de TEPT muy superior a lo esperado, muchos de ellos agrupados en aldeas de damnificados lo que aumenta su vulnerabilidad psicosocial.

Ante el doble problema del aumento de la prevalencia del TEPT y la escasez de recursos de la red de asistencia, surge una oportunidad importante para Universidades y otras instituciones dedicadas a la formación de profesionales en el área de la salud mental. Dicha oportunidad está en orientar las actividades de investigación aplicada al tratamiento de los síntomas del TEPT y otros trastornos relacionados tales como la depresión, ansiedad, angustia y ataque de pánico, abuso de alcohol y drogas, y violencia intrafamiliar (8,31-36). Un paso en ese sentido es el que ha dado la Facultad de Psicología de la Universidad de Talca. Aquí, en el marco del programa de investigación en Calidad de Vida y Ambientes Saludables, estamos trabajando en el tratamiento del TEPT utilizando psicoterapias basadas en la evidencia, justamente en las comunas costeras de la Región del Maule, las zonas más afectadas (7).

Finalmente, quiero terminar con una reflexión respecto de lo qué puede ocurrir si las personas que actualmente están sufriendo de síntomas de TEPT continúan sin tratamientos psicoterapéuticos post-traumáticos eficaces. A la pérdida en la calidad de vida personal, al ausentismo laboral y a la probabilidad de generar otros trastornos graves como la depresión, existe un importante factor genético vinculado con este trastorno: el $41 \%$ de la varianza de los síntomas de TEPT y depresión son explicados por padres o abuelos que hayan sufrido estos trastornos (36). Por lo tanto, si dejamos sin tratamiento a las personas hoy estaremos heredándoles a las futuras generaciones la predisposición de sufrir estos mismos trastornos frente a las catástrofes que de seguro vendrán. Y la falta es doble, debido a que la psicología posee actualmente los conocimientos necesarios para aplicar terapias eficaces en la disminución de síntomas y, por ende, del sufrimiento de las personas 
Agradecimientos: Al Programa de Investigación en Calidad de Vida y Ambientes Saludables de la Facultad de Psicología de la Universidad de Talca.

\section{REFERENCIAS}

1. USGS-United States Geological Survey [Internet]. Tsunami and earthquake research 2010. Disponible en: http://walrus.wr.usgs.gov/tsunami/ Consultado: 14 de julio de 2010.

2. ONEMI-Oficina Nacional de Emergencias [Internet]. Cursos de acción en atención a zonas afectadas 2010. Disponible en: http://reliefweb.int/node/347045 Consultado: 03 de marzo de 2010.

3. Riquelme P. Tres enormes olas devastan Constitución tras sismo. La Tercera 2010; 28 de Febrero. p. 8.

4. INE-Instituto Nacional de Estadísticas. Censo 2003: Síntesis de Resultados. Santiago de Chile: La Nación; 2003.

5. Ministerio del Interior-Gobierno de Chile [Internet]. Fallecidos confirmados con RUT y certificado de defunción de registro civil (actualizado al 15/04/2010). Disponible en: http:// www.interior.gov.cl/ Consultado: 14 de Julio de 2010.

6. Ministerio del Interior-Gobierno de Chile [Internet]. Denuncias por presunta desgracia relacionadas con el terremoto y maremoto del 27 de febrero. Disponible en: http://www.interior.gov.cl/ Consultado: 14 de Julio 2010.

7. Leiva M. Creación de un indicador de impacto de terremotos a partir de datos recogidos en personas que vivieron el 27-F. Salud \& Sociedad. 2010; 1 (3): 178-185.

8. Rodríguez J, Zaccarelli M, Pérez R. Guía práctica de salud mental en situaciones de desastres. Washington DC: PAHO; 2006.

9. Solvason HB, Ernst H, Roth W. Predictors of response in anxiety disorders. Psychiatric Clinics of North America. 2003; 26: 411-433.

10. López-Ibor JJ, Valdés M. DSM-IV. Manual diagnóstico y estadístico de los trastornos mentales. Madrid: Masson; 2008.

11. Pérez C, Vicente B, Zlotnick C, Kohn R, Johnson, J, Saldivia S, et al. Estudio epidemiológico de sucesos traumáticos, trastorno de estrés post-traumático y otros trastornos psiquiátricos en una muestra representativa de Chile. Salud Mental. 2009; 32: 145-153.

12. Wu H, Chou P, Chou F, Su C, Tsai K, Ou-Yang W, et al. Survey of quality of life and related risk factors for a Taiwanese village population 3 years post-earthquake. Australian and New Zealand Journal of Psychiatry. 2006; 40: 355-361.

13. Roussos A, Goenjian A, Steinberg A, Sotiropoulou C, Kakaki M, Kabakos C, et al. Posttraumatic stress and depressive reactions among children and adolescents after the 1999 earthquake in Ano Liosia, Greece. American Journal of Psychiatry. 2005; 162: 530-537.

14. McMillen JC, North C, Smith E. What parts of PTSD are normal: intrusion, avoidance, or arousal? Data from the northridge, California, earthquake. Journal of Traumatic Stress. 2000; 13: 57-79.

15. Dewaraja R, Kawamura N. Trauma intensity and posttraumatic stress: Implications of the tsunami experience in Sri Lanka for the management of future disasters. International Congress Series. 2006; 1287: 69-73.

16. Bland S, Valoroso L, Stranges S, Strazzullo P, Farinaro E, Trevisan M. Long-term follow-up of psychological distress following earthquake experiences among working Italian males: a cross-sectional analysis. J Nerv Ment Dis. 2005; 193(6):420-423.

17. Cairo JB, Dutta S, Nawaz H. The prevalence of posttraumatic stress disorder among adult earthquake survivors in Peru. Disaster Medicine and Public Health Preparedness. 2010; 4: $39-46$.

18. Lai TJ, Chang CM, Connor KM, Lee LC, Davidson, JR. Fulland partial PTSD among earthquake survivors in rural Taiwan. J Psychiatr Res. 2004; 38(3): 313-322. 
19. Sharan P, Chaudhary G, Kavathekar S, Saxena S. Preliminary report of psychiatric disorders in survivors of a severe earthquake. American Journal of Psychiatry. 1996; 153(4): 556-558.

20. John P, Russell S, Russell P. The prevalence of posttraumatic stress disorder among children and adolescents affected by tsunami disaster in Tamil Nadu. Disaster Management \& Response. 2007; 5: 3-7.

21. Figueroa R, González M, Torres R. Plan de Reconstrucción Psicológica Post Terremoto. Revista Médica Chilena. 2010; 138: 920-921.

22. Cougle J, Resnick H, Kilpatrick D. PTSD, depression, and their comorbidity in relation to suicidality: cross-sectional and prospective analyses of a national probability sample of women. Depression and Anxiety. 2009; 26: 1151-1157.

23. Green B, Lindly J, Grace M, Leonard A. Chronic post-traumatic stress disorder and diagnostic comorbidity in a disaster sample. Journal of Nerv Ment Disaster. 1992; 180 (12): 760766.

24. Hamblen J, Norris F, Pietruszkiewicz S, Gibson L, Naturale A, Louis C. Cognitive Behavioral Therapy for Postdisaster Distress: A Community Based Treatment Program for Survivors of Hurricane Katrina. Adm Policy Ment Health. 2009; 36: 206-214.

25. Norris F, Donahue S, Felton C, Watson P, Hamblen J, Marshall R. A psychometric Analysis of Project Liberty's Adult Enhanced Services Referral Tool. Psychiatric Services. 2006; 57 (9): 1328-1334.

26. Norris F, Hamblen J, Brown L, Schinka J. Validation of the Short Posttraumatic Stress Disorder Rating Interview (expanded version, Sprint-E) as a measure of postdisaster distress and treatment need. American Journal of Disaster Medicine. 2008; 3(4): 201-212.

27. Norris F, Rosen C. Innovations in Disaster Mental Health Services and Evaluation: National, State, and Local Responses to Hurricane Katrina (Introduction to the Special Issue). Adm Policy Mental Health. 2009; 36:159-164.

28. Leiva M, Quintana G. Factores Ambientales y Psicosociales Vinculados a Síntomas de Ataque de Pánico Después del Terremoto y Tsunami del 27 de Febrero de 2010 en la Zona Central de Chile. Terapia Psicológica. 2010; 28(2): 161-167.

29. Figueroa R, Marín H, González M. Apoyo psicológico en desastres: Propuesta de un modelo de atención basado en revisiones sistemáticas y metaanálisis. Revista Médica de Chile. 2010; 138: 143-151.

30. Galea S, Nandi A, Vlahov D. The epidemiology of post-traumatic stress disorder after disasters. Epidemiology Review. 2005; 2778-91.

31. Goenjian AK, Steinberg AM, Najarian LM, Fairbanks LA, Tashjian M, Pynoos RS. Prospective study of posttraumatic stress, anxiety, and depressive reactions after earthquake and political violence. American Journal of Psychiatry. 2000; 157(6): 911-916.

32. Campbell D, Felker B, Liu C, Yano E, Kirchner J, Chan D, et al. Prevalence of depression-PTSD comorbilidity: implications for clinical practice guidelines and primary care-based interventions. Journal of General Internal Medicine. 2007; 22: 711-718.

33. Canetti D, Galea S, Hall B, Johnson R, Palmieri P, Hobfoll S. Exposure to prolongued sociopolitical conflict and the risk of PTSD and depression among Palestians. Psychiatry. 2010; 73 (3): 219-231.

34. Davidson JR, Hughes D, Blazer D, George LK. Posttraumatic stress disorder in the community: an epidemiological study. Psychol Med. 1991; 21: 713-721.

35. Eksy A, Braun K, Ertem-Vehid H, Peykerli G, Saydam R, Toparlak D, et al. Risk factors for the development of PTSD and depression among child and adolescent victims following a 7.4 magnitud earthquake. International Journal of Psychiatry in Clinical Practice. 2004; 11 (3): 190-199.

36. Goenjian AK, Noble EP, Walling DP, GoenjianAG, Karayanc IS, Ritchieb T, et al. Heritabilities of symptoms of posttraumatic stress disorder, anxiety, and depression in earthquake exposed Armenian families. Psychiatric Genetics. 2008; 18(6): 261-266. 